\title{
Research and Discussion on Combine Harvester Cleaning Loss Detection Technology
}

\author{
Wang Su-zhen ${ }^{1,2, a}$, Lu Bao-chun ${ }^{1, b}$ \\ ${ }^{1}$ College of mechanical engineering, Nanjing University of Science \& Technology, Nanjing 210019, \\ China \\ ${ }^{2}$ Nanjing research institute for agriculture Mechanization, Ministry of Agriculture, Nanjing 210014, \\ China \\ asophi99@126.com, 'blbcnust@sina.com
}

Keywords: harvester, cleaning loss, signal acquisition technology, signal processing and extraction, blind sources separation(BSS).

\begin{abstract}
Combine harvester is generally used machinery equipment. The design, production and using of combine harvester must ensure grain loss meeting its relevant national indicators of quality and performance. Grain loss mainly come from cleaning parts, so that the research of cleaning loss detection technology is very important. But the combine harvester monitoring system about loss is not universal, basically staying at the research stage in China. Under our existing land conditions, with combine existing holdings, the research value and market prospects are very impressive. From the signal acquisition technology, signal processing and extraction and vibration sensor structure, this article introduces the domestic and foreign harvester method and apparatus for testing the cleaning loss. It Also analyses the problems of existing research technology and methods, including the complex calibration procedure of sensor and detection devices ,not being high detection accuracy and so on. On this basis, the author puts forward the main research directions of cleaning loss detection technology and its development trends in the future. Firstly, the key sensor technologies are developed in depth. Secondly, application of blind sources separation(BSS) and other signal process technology in the grain cleaning loss extraction. Lastly, research on loss mechanism and automatic controlling technology for cleaning part.
\end{abstract}

\section{Introduction}

The combine harvester is currently in widespread use of harvester machinery equipment. The design, production and using of Combine must ensure grain loss meeting its relevant national indicators of quality and performance. So the monitoring of grain loss is very necessary. But monitoring systems for the loss of combine harvesters are not universal, basically staying at the research stage. For combine existing holdings and mechanical working area to further promote the trend, the research value and market prospects are very impressive.

Header, cleaning sieves and strawwalker are always main components which cause the loss of harvesting. A large number of experimental data show that the grain losses mainly comes from the cleaning unit. Cleaning loss rate is one of the key indicators of operating performance. However, because the operation conditions of combine harvester have diversified characteristics, only for a kind of harvesting crops or an area to research loss test device is not practical. It is universal existing that there are mismatches between agricultural machinery and agronomic, which has become the bottleneck of market popularization for loss test device extension. Morever, the working environment is bad, combine harvester grain cleaning detection signal is very weak compared with the vibration noise of harvester, almost submerged in the background noise and interference. there are a lot of straw, weeds and other materials from cleaning sieve. How to separate (or extract) the weak signal of grain cleaning loss from the strong background has always been a difficult topic which the harvester research scientists concern about, and also causes many researchers's interest in both domestic and foreign. 


\section{Research status}

In the last century 60's, many foreign countries have begun to study of combine harvester grain loss monitoring technology. Up to now, America, Holland and other countries have been equipped with the mature loss monitoring device, which can timely remind the driver to obtain the loss level of harvester. But China started relatively late in the aspect, there are no mature products to be introduced in the market. In domestic and foreign, researchers mainly begin to research detecting device and method of cleaning loss from three aspects below: signal acquisition and sensor technologies, signal processing and extraction as well as sensor structure vibration.

\section{Signal acquisition technology}

JOHN DEERE, CASE IH and other foreign companies[1,2] generally install a rectangular or circular iron plate at the end of cleaning sieve, and then set up a piezoelectric ceramic in its central position[3](see Fig.1). When a drop of seed impacting on the iron plate, a mechanical vibration is generated with being converted into a pulse signal by the piezoelectric transducer and signal processing ciruit. Then at last we can obtained grain loss through amplitude discrimination processing. But this method is often limited to the choice of sensor material because different materials are not the same for the vibration maximum amplitude and different grains reflection on the same type of sensors are not also the same. In addition, a single sensor can only make us obtain the grain loss of the effective area.

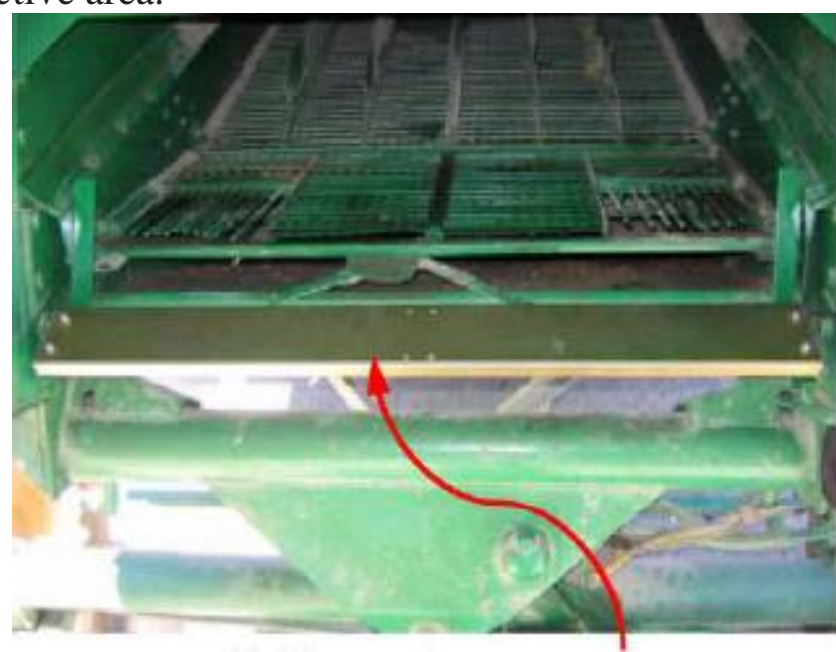

Fig. 1. Upper sieve sensor of John Deere 955

U.S. Patent No. 4,036,065 [4] proposed a method with using voice recognition to monitor grain loss. A high-sensitivity miniature microphone was installed at the tail of cleaning sieve, by picking different values of grain, chopped straw, weed and other material impact on sensor board sound frequency and amplitude to identify them. But the background noise and vibration of harvester itself will produce considerable interference, which makes grain such weak signal difficult to be extracted.

Domestic researches on harvester cleaning loss are rather late compared to with foreign organisms.Based on the digestion and Based of foreign study, the proposed method of indirect getting loss signal was proposed. China Agricultural University, Chen, D., used the experimental method proposed to establish prediction model for a combine harvester between feeding and harvest processing loss with three functions (power, exponential and quadratic). Among them, quadratic function which has highest accuracy, can be used as an effective tool to predict the processing loss[5]. Aiming at a single sensor for only getting local loss signal, Jiangsu University Mao, H. P., etc. proposed to build the piezoelectric crystal vector sensor array to effectively improve the effective area of grain loss detection and accuracy of loss rate [6]. Jiangsu University Zhao, Z.,etc. installed loss sensor in grain separating place, through the establishment of mathematical model of threshing cylinder radial separation for grain loss signal detection[7].

\section{Signal processing and extraction}

In terms of sensor signal processing and extraction, existing grain loss monitor has the basically similar principle[7](see Fig.2). Using a piezoelectric sensor to obtain the mechanical vibration, the actual output is the quantity of electric charge, which is linearly proportional to vibration intensity 
and changes into the amount of voltage after an integral circuit. Because Grain and other materials vibration signals generated by the piezoelectric transducer is very weak, and often submerged in a lot of noise, so the amount of voltage must conduct the first amplification (shown in the circuit magnification of R5/R3) and can be recognized by $\mathrm{MCU}$ or secondary instrument. The signal amplitude and frequency, which these different materials (rice husk, weed stem, grain, etc.) impact on sensor metal plate to produce, are all different. Among them, the grain signal is presented as high frequency and amplitude, and the crushed stem, etc. materials show low frequency and small amplitude. After voltage amplification, the interference signals of broken stem and others are filtered by means of the band-pass filter circuit. Finally, pulse signals are outputted through the voltage limiter circuit, which can directly be detected by SCM. This method needs to determine the frequency and amplitude range of each material in advance. There are too many varied factors, such as crop varieties, throwing height and so on. So that the sensor pre-calibration work is very complicated and must consume the massive manpower. It is also the key technology to testing equipment. Moreover, without considering the influence of the harvester itself work vibration and high frequency noise, this all affect the loss detecting accuracy and reliability.

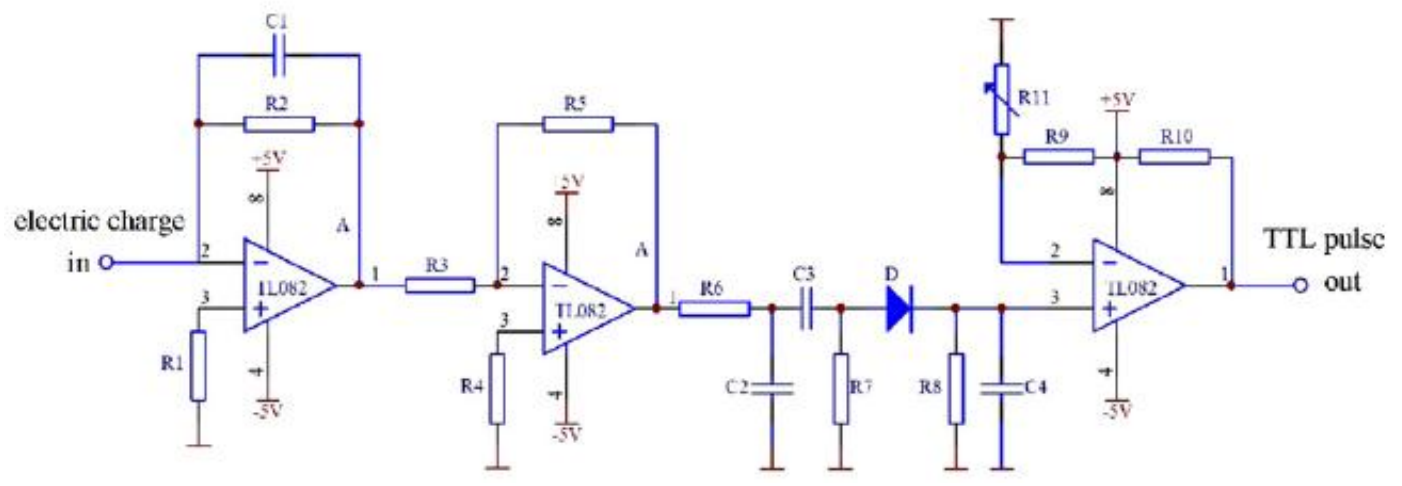

Fig. 2: The basic principle of sensor processing

Processing and extraction of loss signal is the key and the difficult to solve the loss testing accuracy. For Chinese national conditions and the agricultural industry standard, there is some research value. In recent years, in China researchers have undertaken a number of theoretical, simulation and experimental study about cleaning loss testing technology. Under strong noise background on the extraction cleaning grain loss signal, Jiangsu University, Ni, J. in 2010 proposed the use of a Butterworth filter separating vibration noise from grain signal characteristics [8]. Hanping, M., etc. extracted grain loss signal by using symmetrical structure of the sensor with the band-pass filter circuit [9].

In recent years, with the development of signal processing technology and computer technology, the signal extraction aspect has a new breakthrough. Gao, J., etc. of Jiangsu University researched the cleaning loss by making use of chaotic system's characteristic, which is so extremely sensitive to initial conditions and parameters to change the periodic solutions of system[10]. Guan, J.J. [11] and Fang, Y. [12] one after another proposed the use of independent component analysis (ICA) technology to extract grain loss signal of multiple sets of observed sensors. Because this method has no need of prior knowledge, we can dig out the loss grain signal whose amplitude is fastly decayed under the strong background noise. Test has higher precision.

\section{Vibration sensor structure}

In order to avoid interference of harvester itself vibration to the accuracy of sensor signal acquisition, the sensor installation needs to consider the aseismic design. According to the vibration isolation principle and characteristics of rubber isolator low natural frequency and convenient installation, Li, Y.M., etc. of Jiangsu University designed the vibration aseismic structure of cleaning loss sensor to isolation effect of the vibration and noise of the harvester on the loss sensor[13].

\section{Reflections}


The researches on cleaning loss technology in home and abroad have made great progress. Especially in some foreign harvesters cleaning loss detecting devices have become quite common. As China's land situation, I believe that in China there are still a lot of research space and requirement, cleaning loss test techniques to the following questions will be further attention:

\section{Calibration of sensor and detection device}

To improve the utilization rate of agricultural machinery and increase the economic benefits of machine driver, harvester machinery are now mostly designed for multifunctional type. A large number of experimental studies show that for rape, rice and other crop grain from the same height to loss sensor, their vibration amplitudes are different. Not only that, for the same kind of crop varieties, their amplitudes of vibration are not the same, and their vibration frequency of crops from different height are also different. So with the external factors in change such as the harvest crops, varieties, environment, etc., farmers often need to be re-calibration work on automatic test equipment and loss sensor. Existing calibration work is tedious and professional and technical requirements are high, thus increasing the difficulty and workload of harvest operations and affecting seasonal rush in the harvest. Studying on a set of agricultural machinery and agronomic automatic calibration method to replace the existing conventional manual calibration method is one of the key technologies of generalized loss test device.

\section{To improve the measurement accuracy}

Based on Chinese national conditions of existing rural land, many farmers during the harvest can not accept the grain loss phenomenon. Simply according to upper limit setting of the loss alarm for reminding machine driver, it is not able to meet the demand of peasants. So the loss detection accuracy has certain requirement. But mature loss devices of overseas generally separate signals according to the different frequency and amplitude of signals which are produced by material impact on sensor plate. Many import harvesters have loss detecting device, but in practical application, the domestic machine and farmers often remove them, mainly because of detection accuracy and testing complexity.

\section{Research trends}

\section{Research on the sensor key technology in depth}

Sensor key technology of cleaning loss includes sensor structure design, sensor test methods, sensor detection materials, distribution of the cleaning loss signals, and sensor calibration methods, etc. Liming, Z., etc.[14] used PVDF piezoelectric film as sensor array material, combined with the size of grain loss sensor to obtain the spatial distribution, and then applied the diode envelope detection signal processing for effective grain. This sensor detection method, to some extent, can improve the measurement accuracy and response speed. Aiming at sensor calibration of different operating conditions demand, Zhan, L. Y. L. Z. Z., etc. [15] designed grain loss monitor sensor calibration test bench with consisting of the lifting platform, lifting drives, feeding device, and sensor mounting platform. For the analysis of grain loss monitor sensor sensitive plate structure influencing on grain collision signal, Liang, Z., etc. [16] researched the direct relation between the sensitive plate vibration characteristics and grain loss monitor sensor detection performance on the base of modal analysis, in order to improve the sensor detection frequency providing technical support.

The above researches are fundamental and essential, but this is not enough. Only on the basis of satisfying the agronomy demands, can the loss detection research be significant.

\section{Application of BSS and other advanced signal technology}

BSS technology has solved many problems in the fields of application.At present, it has been successfully applied to the separation and recognition of speech signals, data communication and array signal processing, image processing and recognition, geo spatial information processing, biomedical signal processing and many other fields. When the hardware measures can not further solve the problem of testing device, including improving accuracy, sensor loss detection automatic calibration problem,et al,based on modern signal processing and computer technology, developing advanced signal technique of the BSS is an inevitable research trend. 
Loss signal detection is actually a similar "cocktail party" BSS problem. BSS method, under the condition of the source signal and the system transfer function being unknown, separates independent source signals from a group of mixed-signal measured by the sensors. When it is difficult to establish the mathematical model from the signal source to the sensor or transfer prior knowledge can not be obtained, the blind signal separation is a better research method. General BSS model[17](see Fig.3) where $X$ denotes $m$ dimensional observation vector, $S$ denotes $n$ dimensional signal vector, and $A$ denotes $m \times n$ dimensional signal matrix. The destination of BSS is to obtain a separation matrix $W$ through $X$, the signal $Y$ obtained with $W$ on $X$ is the optimal estimation of the source signal $S$, and their relationship can be expressed as: $Y=W X=W A S$.

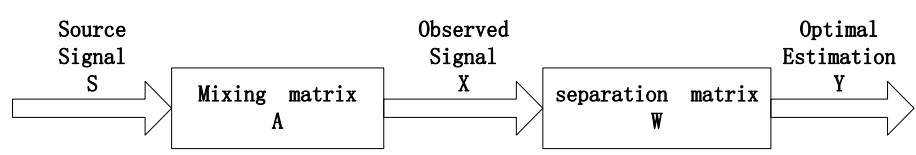

Fig.3:General BSS model

Among them, the independent analysis (ICA) is the most commonly used method of blind source separation technology. ICA technology and the application range are very wide, overcoming the problem in many application fields[18]. The existing research on the loss signal based on ICA method still remains in the theory of FASTICA algorithm according to maximum entropy of the objective function. It is the Newton iteration for a set of given data of recursive calculation, not only belonging to the batch processing mode,but also the separating matrix existing uncertain initial value and cumulative iteration error[19]. The problem still exists in practical application. But in the design of loss test device, the independent components are only online extracted. In the future, we still need to study optimization algorithm for a single sample with data obtained for continuously updating separation matrix parameters.

In addition, according to the nature of grain vibration function, other advanced signal processing methods will be applied to the study of cleaning loss detecting technology, in order to simplify the work through the method of signal processing, reduce cost of testing device, increase the extraction precision of the grain loss, and improve the quality of work and efficiency of the harvester,with taking agricultural machinery and agronomic matching as criteria.

\section{Loss Mechanism and Control Technology Research}

Just testing the grain amount and rate of cleaning loss is a passive grain loss monitor, because the machine can not know the specific reasons for impacting on the work quality[20]. Separating and cleaning of harvesting machinery are nonlinear and complex, both to ensure grain separating and cleaning to achieve a certain purity, and to ensure the minimum grain loss. This involves the airflow dynamics analysis of the cleaning fan, kinematic analysis of grain above vibration sieve and MOG thrown, and the interaction studying among them. Only by learning the important factors of the grain cleaning loss generated, can harvester machinery parts be fundamentally correctly controlled for reducing the loss rate and improving the quality of work.

However, with the change of soil, climate, crop types, operating conditions, etc., in order to reduce loss, machine drivers need to operate machinery by experience for changing work parameters so as to adapt to changes in load, which leads to the driver's labor intensity very large. So by the guide of the loss theory, it is very important to study on the separation and cleaning loss parameter's automatic controlling strategy and system. And at the same time combined with electromechanical integration technology to research harvester automatic adjustable structure, it is harvester development trend to improve the machine driver's comfortably. In recent years, in the abroad, already study according to the loss of signal feedback how to automatically control parameters about separating and cleaning such as cleaning loss, tail sieve opening, fan speed and so on. In the aspect, many researchers have made important contributions. Hall in 1992[21] identified and controlled the threshing cleaning loss parameters for real-time operation parameter and environment by using the neural network model trained, but this method is not practically strong because it needs the more neuron number with the higher requirements for storage and computing power. Diekhans in 2001[22] built the separating and cleaning operation parameter database which the measured operating parameters are comparable to in the controlling system for adjusting 
operation parameters. The database can be artificially added by operating the device. This method is simple, stronger practical, and easy to operate. In 2008, Omid, M. [3] used fuzzy logic controller (FLC) method combined with expert system for automatic for controlling cleaning loss. This method has the space and value for further research. But the current domestic research on this aspect is still blank. With the accelerated in recent years in the land transfer process, it is urgent for the automatic controlling technology research and market promotion.

\section{Conclusions}

As one of the harvester detection criteria, cleaning loss detecting technology research has a broad market prospect. This paper describes the current research status of foreign and domestic harvester about cleaning loss monitoring technique, points to requirements of the current China land conditions for cleaning loss monitoring technology, sums up key technologies of cleaning loss research, and expects its development prospects.In the future, we need continue to study about sensor key technology. Moreover, focusing on application of BSS and other advanced signal processing technology, the goal is that the software approach can solve some long-term problems, for example, which loss sensor can not do auto calibration work, improving the extraction precision of the grain loss and the quality of work and efficiency of the harvester, and so on. What's more, we should be more positive about loss mechanism and control technology research.

\section{Acknowledgements}

This work is supported by the Science and Technology Support Program of Jiangsu Province No. BE2012313.

\section{References}

[1] Diekhans, N., \& Behnke, W., U.S. Patent No. 4,902,264. Washington, DC: U.S. Patent and Trademark Office, 1990.

[2] Behnke, W., Hubner, R., Damm, W., Muller, H., \& Bernhardt, G. , U.S. Patent No. 6,146,268. Washington, DC: U.S. Patent and Trademark Office,2000.

[3] Omid, M., Lashgari, M., Mobli, H., Alimardani, R., Mohtasebi, S., \& Hesamifard, R., Design of fuzzy logic control system incorporating human expert knowledge for combine harvester. Expert Systems with Applications, 37(10), pp.7080-7085, 2010.

[4] Van Quekelberghe, E. P., U.S. Patent No. 6,524,183. Washington, DC: U.S. Patent and Trademark Office,2003.

[5] Chen, D., Wang, S., Kang, F., Zhu, Q., \& Li, X., Mathematical model of feeding rate and processing loss for combine harvester. Transactions of CSAE,27(9),pp.18-21,2011.

[6] Mao, H. P., \& Ni, J., Finite element analysis and measurement for array piezocrystals grain losses sensor. Transactions of the Chinese Society for Agricultural Machinery, 39(12), pp.123126, 2008.

[7] Zhao, Z., Li, Y., Chen, J., \& Xu, J., Grain separation loss monitoring system in combine harvester. Computers and Electronics in Agriculture, 76(2), pp. 183-188, 2011.

[8] Ni, J., Mao, H. P., Zhang, X. D., \& Chen, X. H., Application of butterworth filter for testing grain cleaning loss. Transactions of the Chinese Society for Agricultural Machinery,41(6),pp.172-176,2010. 
[9] Hanping, M., Wei, L., Lühua, H., \& Xiaodong, Z.,Design of intelligent grain cleaning losses monitor based on symmetry sensors. Transactions of the Chinese Society of Agricultural Engineering,28(7),pp.34-39,2012.

[10] Gao, J., Zhang, G., Yu, L., \& Li, Y., Chaos detection of grain impact at combine cleaning loss sensor. Transactions of the Chinese Society of Agricultural Engineering,27(9),pp.22-27,2011.

[11] Guan, J.J., The detection of lost grain signal in combine harvester based on ICA. Master thesis, Jiangsu University, 2011.

[12] Fang, Y., The research of blind signals separation algorithm on detection of lost grain in combine harvester. Master thesis, Jiangsu University, 2013.

[13] Li, Y.M., Chen, Y., Zhao, Z., Xu, L.Z., Monitoring method and device for cleaning loss of combine harvester. Transactions of the Chinese Society for Agricultural Machinery, 44(z2),pp.7-11, 2013.

[14] Liming, Z., Xiaochao, Z., \& Yangchun, L., Design of PVDF sensor array for grain loss measuring. Transactions of the Chinese Society for Agricultural Machinery, 41(6), pp.167-171, 2010.

[15] Zhan, L. Y. L. Z. Z., \& Zhong, X. L. C. Y. T., Calibration Experiment of Grain Loss Monitoring Sensor in Combine Harvester. Transactions of the Chinese Society for Agricultural Machinery, S1,2012..

[16] Liang, Z., Li, Y., Zhao, Z., \& Chen, Y., Structure optimization an performance experiment of grain loss monitoring sensor in combine harvester in using modal analysis. Transactions of the Chinese Society of Agriclutural Engineering, 29(4),pp.22-29,2013.

[17] Yu, X.C., Blind source separation theory and Application, Beijing:Science Press,2011.

[18] Yang, J. M., Yu, H., \& Wei, G., Independent component analysis and its application to signal processing. Journal of South China University of Technology(Natural Science Edition), 40(11),pp. 1-12, 2012.

[19] Xiao-niu(2), Y. J. L. Y., \& Han, L. J. D. Z. Y., Wei-jia(1) (1) (State Key Lab. of Integrated Service Networks, Xidian University, Xi'an 710071, China) (2) (No. 36 Research Institute of China Electronics Technology Group Corporation, Jiaxing 314001, China); A Multi-stage FastICA Algorithm to Remove Error Propagation [J]. Journal of Electronics \& Information Technology, 11, 2009.

[20] Jie, Z., Prospect of test method of grain random rate in China. Agricultural Mechanization Research, 7, pp. 5-9, 2009.

[21] Hall, J. W. , Emulating human process control functions with neural networks. Doctoral dissertation, University of Illinois at Urbana-Champaign, 1992.

[22] Diekhans, N., U.S. Patent No. 6,205,384. Washington, DC: U.S. Patent and Trademark Office, 2001. 